Bull. Austral. Math. Soc.

VOL. $60(1999) \quad[55-65]$

\title{
ORDER PRESERVING FUNCTIONS ON ORDERED TOPOLOGICAL VECTOR SPACES
}

\author{
J.C. Candeal, E. Induráin and G.B. Mehta
}

\begin{abstract}
In this paper we prove the existence of continuous order preserving functions on ordered topological vector spaces in an infinite-dimensional setting. In a certain class of topological vector spaces we prove the existence of topologies for which every continuous total preorder has a continuous order preserving representation and show that the Mackey topology is the finest topology with this property. We also prove similar representation theorems for reflexive Banach spaces and for Banach spaces that may not have a pre-dual.
\end{abstract}

\section{INTRODUCTION}

In this paper we prove the existence of continuous order preserving functions on ordered topological vector spaces. The emphasis in the literature has been on proving the existence of continuous order preserving functions on general preordered topological spaces and on subsets of finite-dimensional Euclidean spaces. There are only a few wellknown results (see $[19,23]$ ) on the existence of continuous order preserving functions on infinite dimensional linear spaces. For some recent work in this field the reader is referred to $[\mathbf{8}, \mathbf{1 8}]$. The object of this paper is to partially fill this gap in the literature by proving some new results on the existence of continuous order preserving functions on totally preordered topological vector spaces.

We first prove in Theorem 1 that if $E$ is a topological vector space and there is a separable topological vector space $F$ such that $E$ is the topological dual of $F$ then there exists a topology $t$ on $E$ such that every $t$-continuous total preorder on $E$ has a continuous order preserving representation. We also show that the Mackey topology of the duality $(E, F)$ is the finest compatible linear topology on $E$ that has this property. A similar result is shown to hold for norm-separable Banach spaces.

On the other hand, the norm topology of a Banach space $E$ need not have the property that every norm continuous total preorder has a continuous representation. To show this we give an example of a norm-continuous total preorder on a Banach lattice that is not representable. Furthermore, it should be noted that in [13] it is proved that there is a continuous non-representable total preorder on any non-separable metric

Received 9th December, 1998

Copyright Clearance Centre, Inc. Serial-fee code: 0004-9729/99 \$A2.00+0.00. 
space. In view of these considerations, it follows that if we want a general result about representability in an infinite-dimensional context we need to assume stronger properties on both the Banach space and the preorder.

Accordingly, our first result in this direction involves a Banach space $E$ that is reflexive. We prove in Theorem 2 that in a reflexive Banach space with a countable coinitial subset every continuous convex total preorder has a continuous quasi-concave order preserving representation. The proof of this theorem is based on the Arrow-Hahn method [3, pp.82-87] which consists in defining an order preserving function in terms of the given metric on the Banach space $E$.

In each of the preceding two theorems the topological vector space $E$ has a predual. Suppose now that $E$ is a Banach space that may not have a pre-dual such as the classical sequence space $c_{0}$ or the Lebesgue space $L^{1}([0,1], \mu, \mathbb{R})$. Then the methods used in Theorems 1 and 2 are not applicable because they depend upon properties of the weak*-topology. Clearly, in order to get some results in this general context one needs strong (and somewhat different) assumptions on the preorder. In Theorem 3 we present a set of sufficient conditions for the existence of a continuous order preserving function on a Banach space $E$ that need not have a pre-dual. Specifically, we assume that the preorder is convex and has locally compact upper sections. This theorem partially answers a question raised in our earlier paper [8, p.245].

In conclusion, it may be noted that infinite-dimensional linear spaces of the type studied in the paper are now commonly encountered in mathematical economics and related fields. See $[\mathbf{1}, \mathbf{2}, \mathbf{4}, \mathbf{6}, \mathbf{2 1}]$.

\section{Preliminaries: Definitions and Notations}

Let $E$ be a topological vector space and $\preceq$ a total preorder on a subset $X$ of $E$. The relation $\preceq$ on $X$ may be interpreted as a weak preference relation on $X$ so that $x \preceq y$ means that $y$ is at least as good as $x$. The relation $\preceq$ on $X$ induces two new relations on $X$ as follows. If $x, y \in X$ then define $x \prec y$, if and only if $x \preceq y$ and $\neg(y \preceq x)$. This relation on $X$ is irreflexive and transitive and may be interpreted as a strict preference relation on $X$. If $x, y \in X$ then define $x \sim y$, if and only if $x \preceq y$ and $y \preceq x$. This relation on $X$ is an equivalence relation on $X$ and may be interpreted as an indifference relation. For each $x \in X$, the $\sim$-equivalence class of $x$ is denoted by $I(x)$.

Let $t$ be a topology on a topological vector space $E$ and $\preceq$ a total preorder on a subset $X$ of $E$. Then $\preceq$ is said to be a $t$-upper-continuous if for each $y \in X$ the set $\{x \in X: y \preceq x\}$ is $t$-closed in $X$ and $t$-lower-continuous if for each $y \in X$ the set $\{x \in X: x \preceq y\}$ is $t$-closed in $X$. The relation $\preceq$ is said to be $t$-continuous if it is both upper and lower continuous with respect to the topology $t$. 
Let $\preceq$ be a preorder on a set. The upper section $\{x \in X: y \preceq x\}$ of $y$ is denoted by $C(y)$ and the lower section $\{x \in X: x \preceq y\}$ of $y$ is denoted by $W(y)$.

Let $\preceq$ be a total preorder on a set $E$. A subset $Z$ of $E$ is said to be Debreu orderdense if for every pair $(x, y) \in E$ with $x \prec y$ there exist $z \in Z$ such that $x \preceq z \preceq y$. Then $(E, \preceq)$ is said to be Debreu order separable if there is a countable subset $Z$ that is Debreu order-dense in $E$.

Let $\preceq$ be a total preorder on a subset $X$ of a topological vector space $E$. Then $\preceq$ is said to be convex if for each $x \in X$ the upper section $C(x)$ is a convex set.

Let $K$ be a subset of a normed linear space $E$. Then $K$ is said to be proximinal in $E$ if for each $x \in E$ there exists $y \in K$ such that $\|x-y\| \leqq\|x-z\|$ for all $z \in K$.

Let $(E, \preceq)$ be a preordered topological vector space. The preorder $\preceq$ has a countable co-initial subset $Z$ if $Z$ is countable and for each $y \in E$ there exists $x \in Z$ such that $x \preceq y$.

If $E$ is a normed linear space, the closed ball of radius $r>0$ around a subset $K$ of $E$ will be denoted by $B(K, r)$.

Let $(E, F)$ be a dual pair. Then the polar topology on $E$ determined by the class of finite subsets of $F$ is called the weak topology on $E$ and is denoted by $w(E, F)$ or $w$. If $E$ has a pre-dual $F$, the $w(E, F)$ topology on $E$ is also called the weak-star (weak*) topology. The polar topology on $E$ determined by the class of absolutely convex $w(F, E)$-compact subsets of $F$ is called the Mackey topology on $E$ and is denoted by $m(E, F)$.

Let $\preceq$ be a total preorder on a topological space $E$. Then a real-valued function $f$ on $E$ is said to be order-preserving if $x \preceq y$ implies that $f(x) \leqq f(y)$ and $x \prec y$ implies that $f(x)<f(y)$. A utility function on $(E, \preceq)$ is a real-valued order preserving function.

Following [15], we now define the concept of a useful topology on a set.

Let $E$ be a set. Then a topology $t$ on $E$ is said to be useful if every $t$-continuous total preorder $\preceq$ on $E$ can be represented by a $t$-continuous order preserving realvalued function.

\section{EXISTENCE OF ORDER PRESERVING FUNCTIONS}

We now prove the following theorem.

THEOREM 1. Let $E$ be the dual of a separable locally convex topological vector space $F$ and assume that $E$ is $\sigma$-compact in the weak* topology. Let $X$ be a weak* closed convex subset of $E$ and suppose that $t$ is a topology on $E$ that is coarser than or equal to the Mackey topology $m(E, F)$. Then every $t$-continuous total preorder admits a $t$-continuous real-valued order preserving representation. 
Proof: Recall that, by the Mackey-Arens Theorem [20, p.205], the finest linear topology on $E$ compatible with the dual pair $(E, F)$ is the Mackey topology. The strategy of the proof is to show that $X$ is a separable topological space with respect to the induced Mackey topology on $X$. Then $X$, being a convex subset, is a connected and separable topological space. Therefore Eilenberg's Theorem [12] applies. In order to see this let us first prove that $X$ is $w^{*}$-separable.

Let $E=\bigcup_{n=1}^{\infty} K_{n}$ where for each $n=1,2, \ldots, K_{n}$ is compact in the weak* topology of $E$. Since $E$ is the dual of the separable topological vector space $F$, the set $K_{n}$ is metrisable in the weak* topology $[22$, p.70]. Since any compact metric space is separable $[11, \mathrm{p} .233]$, we may conclude that for each $n, K_{n}$ is a separable topological space in the weak* topology. So $E$ is separable in this topology and therefore, $X$, being $w^{*}$-closed, is also a $w^{*}$-separable topological space.

Denote by $D=\left(d_{n}\right)_{n=1}^{\infty}$ a $w^{*}$-dense subset of $X$. Let us now prove that $X$ is also $m(E, F)$-separable. Consider the Mackey-closed linear subspaces $\vee D^{-}, \vee X^{-}$ generated by $D$ and $X$ respectively, that is, $\vee D=\left\{\sum_{i=1}^{m} \lambda_{i} d_{i}: \lambda_{i} \in \mathbb{R}, d_{i} \in D\right\}$ and $V X=\left\{\sum_{i=1}^{r} \alpha_{i} x_{i}: \alpha_{i} \in \mathbb{R}, x_{i} \in X\right\}$, the bar denoting the closure in the Mackey topology.

Two cases may occur: either $\bigvee D^{-}$is a proper linear subspace of $\bigvee X^{-}$or $\bigvee D^{-}=$ $V X^{-}$. Let us see that the first situation leads to a contradiction. Indeed, by the HahnBanach Theorem $[22$, p.60] there is a Mackey-continuous linear function $g: E \rightarrow \mathbb{R}$ such that $g \mid \vee_{D^{-}} \equiv 0$ and $g \mid \vee_{X^{-}} \neq 0$. But this is impossible because $g$ is also $w^{*}$ continuous, and hence, so is its restriction to $\vee X^{-}$, and because $D \subseteq X$ is $w^{*}$-dense in $X$, so that $\bigvee D^{-}$is $w^{*}$-dense in $\bigvee X^{-}$.

In the second case, consider the set $B=(\bigvee D \cap X)^{-}$(closure in the Mackey topology). Note that, since $D \subseteq X$ and $X$ is a convex $w^{*}$-closed (hence Mackeyclosed) subset, $B$ is a nonempty convex and Mackey-closed subset of $E$ contained in $X$. Let us see that $B=X$. Indeed, otherwise $B$ would be a proper subset of $X$. So there would exist $a \in X \backslash B$. Than again by the Hahn--Banach Theorem there is a Mackey-continuous linear function $f: E \rightarrow \mathbb{R}$ and $\gamma \in \mathbb{R}$ in such a way that $f(b) \leqslant \gamma, \forall b \in B$ and $f(a)>\gamma$. But this is not possible since $f$ is also $w^{*}$-continuous, hence its restriction to $X$, and $D \subseteq B$ is $w^{*}$-dense in $X$. Therefore $B=X$. So $B$ being the Mackey closure of $\bigvee D \cap X$ is Mackey separable. Notice on this point that

$\left\{\sum_{i=1}^{m} q_{i} d_{i}: d_{i} \in D\right.$ and $q_{i}$ is a rational number for $\left.I=1, \ldots, m\right\} \cap X$ is dense in $X$. 
REMARKS.

1. If $F$ is metrisable, in particular a normed space, then the condition of $E$ being $\sigma$-compact in the weak* topology is always satisfied.

2. For a topology $t$ coarser than or equal to the weak* topology the separability of $F$ can be dispensed with. In this case, every $t$-continuous total preorder is countably bounded and, therefore, has a $t$-continuous order preserving representation by a result of Monteiro [19, Example 4 and Theorem 3].

Corollary 1. Let $E$ be a Banach space with a separable pre-dual and $O \subseteq$ $E$ a Mackey-open and convex subset. Then the finest locally convex topology on $E$ compatible with the dual pair $(E, F)$ that induces on $O$ a useful topology is the Mackey topology.

Proof: By taking $X=E$ in the statement of Theorem 1 and following its proof we see that $E$ is a Mackey-separable topological space. Now since $O$ is a Mackey-open subet of $E$ it follows that the separability property is inherited by $O[\mathbf{1 1}, \mathrm{p} .175]$. Since $O$ is connected (it is a convex set) and separable the conclusion follows again from Eilenberg's Theorem.

Since the Mackey topology and the norm topology, which is finer, may not coincide it is clear that, in general, the norm topology on a Banach space cannot be expected to be useful. Actually, even with the additional requirement of being convex, a normcontinuous total preorder could still fail to be representable as the next example shows. This example is based on Monteiro [19] and is esentially an extension of an example of Fishburn [14].

EXAMPLE 1 . Let $Y=[0, \Omega), \Omega$ being the first uncountable ordinal. For a given $\alpha \in Y$ we will denote by $\delta_{\alpha}$ the caracteristic function of $\alpha$. We consider the space $l^{1}(Y)$ defined as follows:

$$
l^{1}(Y)=\left\{\sum_{\alpha \in Y} \lambda_{\alpha} \delta_{\alpha}: \lambda_{\alpha} \in \mathbb{R} \mid \text { and }\left|\sum_{\alpha}\right| \lambda_{\alpha} \mid<\infty\right\} .
$$

It is clear that $l^{1}(Y)$ is a dual Banach lattice. Let $\ell_{+}^{1}(Y)$ be the positive cone of $1^{1}(Y)$, that is $\ell_{+}^{1}(Y)=\left\{\sum_{\alpha \in Y} \lambda_{\alpha} \delta_{\alpha}: \lambda_{\alpha} \geqslant 0\right.$ and $\left.\sum_{\alpha} \lambda_{\alpha}<\infty\right\}$ and consider $X=\ell_{+}^{1}(Y)-\{0\}$. Claim. There is a norm-continuous and convex total preorder defined on $X$ which is not representable by a utility function.

Proof: Let $Z$ be the positive unit sphere of $l^{1}(Y)$, that is $Z=\left\{\sum_{\alpha \in Y} \lambda_{\alpha} \delta_{\alpha}: \lambda_{\alpha} \geqslant\right.$ 0 and $\left.\sum_{\alpha} \lambda_{\alpha}=1\right\}$. By $[19$, Theorem 6$]$ there exists a norm-continuous, convex and non- 
representable total preorder defined on $Z$. Denote this preorder by $\preceq$. Then we define on $X$ the following total preorder $\preceq^{*}: x \preceq^{*} y$ if and only if $x /\|x\| \preceq y /\|y\|(x, y \in X)$.

Clearly $\preceq^{*}$ is well defined and it is norm-continuous. In order to see the convexity notice that the norm is additive on $X$.

Observe also that the preorder $\preceq^{*}$ is homothetic, that is $x \preceq^{*} y$ if and only if $\lambda x \preceq \preceq^{*} \lambda y, \forall \lambda>0$. Thus we obtain an infinite-dimensional example of a continuous and homothetic preorder which is non-representable (see [7]).

The previous example shows that in convex cones of abstract Banach spaces (even dual spaces) there are continuous, convex and homothetic total preorders which are non-representable. Moreover, in a recent paper Estévez and Hervés [13] have proved that in every non-separable metric space there exists a continuous and total preorder which is not representable by an order preserving function. Thus if we want to obtain a general result of representability in an infinite-dimensional context we need to assume nicer properties for both the Banach space and the preorder.

The next theorem states that over the class of convex preorders defined on normclosed and convex subsets of reflexive Banach spaces with a countable co-initial subset the norm topology is useful. The proof given below consists in showing that there exists a countable order-dense subset and then applying [10, Lemma 2]. It should be noted that our next theorm is not a corollary of Theorem 1 because if a Banach space $E$ with a separable pre-dual is reflexive then it is separable and we do not demand this at all.

Theorem 2. Let $E$ be a reflexive Banach space and $X \subseteq E$ a norm-closed convex subset. Suppose that $\preceq$ is a convex and norm-continuous total preorder on $X$, having a countable co-initial subset. Then $\preceq$ is representable by a continuous (and quasi-concave) order preserving function.

Proof: For each $x_{0} \in X$ by using the Arrow-Hahn approach [3, pp.82-87], we are going to construct an order preserving function over the upper section $C\left(x_{0}\right)$ of $x_{0}$. For every $x \in C\left(x_{0}\right)$, we define $U(x)$ to be the distance from $C(x)$ to $x_{0}$. Let us see that this function is well-defined. Indeed, since $\preceq$ is convex and continuous and $E$ is reflexive it follows that each upper section $C(x)$ is a $w^{*}$-closed convex subset of $E$. Now each $w^{*}$-closed subset of a Banach space with a predual is proximinal [16, p.116]. Therefore the function $U$ is well-defined.

Arguing as in Arrow-Hahn [3, pp.83-84] one can see, since $\preceq$ is continuous, that $U(x)$ is an order-preserving function on $C\left(x_{0}\right)$. Therefore, there is a countable subset that is Debreu order-dense in $C\left(x_{0}\right)$ [5, Theorem 1.4.8].

Let now be $\left\{z_{1}, z_{2}, \ldots, z_{n}, \ldots\right\}$ a countable co-initial subset. Then by the first part of the proof for every $n \in \mathbb{N}$ there is a countable subset $Z_{n}$ which is Debreu order-dense in $C\left(z_{n}\right)$. Clearly, since $X=\bigcup C\left(z_{n}\right)$ we conclude that the set $Z=\bigcup Z_{n}$ 
is a countable dense subset of $E$ whence $E$ is Debreu order-separable. Now since the preorder is continuous we see that Lemma 2 of Debreu [10] implies that there is a continuous order-preserving function $f$ on $E$. Finally, the function $f$ is quasi-concave because the preorder is convex.

REMARK 3. This last result generalises one due to Shafer [23] for normed linear spaces with a $\preceq$ - first element. Notice also that Monteiro's example [19, Example 4, p.151] is not complete because it is not clear that the preorder is continuous in the weak topology as the next example shows.

EXAMPLE 2. We now give an example of a norm-continuous real-valued concave function $U: l^{2}(\mathbb{N}) \rightarrow \mathbb{R}$ defined on the Hilbert space $l^{2}(\mathbb{N})$ in such a way that $u$ is not weakly lower-continuous. Thus the preorder induced by this order preserving function is a convex and continuous preorder, defined on a reflexive Banach space, having a countable co-initial subset. This shows that Theorem 2 is not a consequence of Monteiro's result $[19, \mathrm{p} .150]$.

In order to construct such an example, let $\left(f_{n}\right)_{n=1}^{\infty}$ be a family of real-valued functions $f_{n}: \mathbb{R} \rightarrow \mathbb{R}$, satisfying the following conditions:

(i) For each $n \in \mathbb{N}, f_{n}$ is an increasing and concave function such that $f_{n}(0)=0$.

(ii) There is $c \in \mathbb{R}, 0<c<1$ such that $\left(f_{n}\right)_{n=1}^{\infty}$ is uniformly bounded on $(-c, c)$.

(iii) $f_{n+1}(-1)<2 f_{n}(-1)$, for every $n \in \mathbb{N}$.

Now define the function $u: l^{2}(\mathbb{N}) \rightarrow \mathbb{R}$ by:

$$
\text { if } x=\left(x_{n}\right)_{n=1}^{\infty} \in l^{2}(\mathbb{N}) \text { then } u(x)=\sum_{n=1}^{\infty} \frac{1}{2^{n}} f_{n}\left(x_{n}\right) \text {. }
$$

Notice that $u$ is well-defined. Also it is easy to see that $u$ is norm-continuous and concave. Let us see that it is not lower-continuous in the weak topology.

In order to do that, let $\left(x^{m}\right)_{m=1}^{\infty} \subset l^{2}(\mathbb{N})$ be the sequence defined by $x^{m}=\left(x_{n}^{m}\right)_{n=1}^{\infty}$ where $x_{n}^{m}=-1$ if $n=m$ and $x_{n}^{m}=0$ otherwise.

Since for every $m \in \mathbb{N}, u\left(x^{m}\right)=\left(f_{m}(-1)\right) / 2^{m}$, it follows by (iii) that $u\left(x^{m+1}\right)<$ $u\left(x^{m}\right)$. That is, $\left(u\left(x^{m}\right)\right)_{m=1}^{\infty}$ is a decreasing sequence of reals. Also, by (i), notice that $u\left(x^{1}\right)<u(0)=0$.

Now take $\alpha=u\left(x^{1}\right)$ and consider the set $F=u^{-1}(-\infty, \alpha]$. By the remarks above it follows that $x^{m} \in F$ for all $m \in \mathbb{N}$. Further it is obvious that the sequence $\left(x^{m}\right)_{m=1}^{\infty}$ converges to 0 in the weak topology. But $0 \notin F$. Thus $F$ is not a weakly closed subset of $l^{2}(\mathbb{N})$ and therefore $u$ is not lower-continuous in the weak topology. 
A family of real-valued functions satisfying conditions (i), (ii) and (iii) is given below. Define $f_{n}(x)$ by:

$$
f_{n}(x)=\left\{\begin{array}{l}
\frac{n+1}{n} x \text { if } x \geqslant-\frac{1}{2} \\
3\left(2^{n}-1\right) x+\frac{1}{2}\left(3\left(2^{n}-1\right)-\frac{n+1}{n}\right) \text { otherwise }
\end{array}\right.
$$

We now turn to the problem of proving the eixstence of a continuous order preserving function on a Banach space $E$ that may not have a pre-dual. As an example of such a space, we may mention the classical sequence space $c_{0}$ which is the space of all sequences of reals that converge to zero. The Banach-Alaoglu and Krein-Milman Theorems imply that the space $c_{0}$ does not have a pre-dual because its closed unit ball has no extreme points (see [25, p.184 and p.204]). As another example of such a space we mention the Lebesgue space $L^{1}([0,1], \mu, \mathbb{R})$ where $\mu$ denotes Lebesgue measure.This space does not have a pre-dual by Gelfand's Theorem [16, p.218]. It is clear, therefore, that one will need strong assumptions on the preorder to get a positive result for such spaces.

THEOREM 3. Let $E$ be a Banach space and $X$ a norm-closed convex subset of $E$. Suppose that $\preceq$ is a norm-continuous total preorder on $X$ such that the following conditions are satisfied:

(1) $\preceq$ is convex:

(2) $\preceq$ has a countable co-initial subset $Z$;

(3) For each $x \in X$, the upper section $C(x)$ is locally compact.

Then there exists a continuous order preserving function on $X$.

Proof: Let $x_{0}$ be an arbitrary but fixed point of $X$. We claim that for each $x \in C\left(x_{0}\right)$ the set $C(x)$ is proximinal with respect to $\left\{x_{0}\right\}$. Indeed, for each real number $s$, consider the set $K_{s}=\left(x_{0}+s B(0,1)\right) \cap C(x)$. For large $s$ this set is nonempty. Now $K_{s}$ is a closed convex set because it is the intersection of two closed convex sets. In particular, $K_{s}$ is a closed subspace of the locally compact space $C(x)$. Hence, $K_{s}$ is locally compact. Since $K_{s}$ is a subset of a translate of a ball of radius $s$ around the origin it follows that $K_{s}$ does not contain any half-line. Now a closed, convex and locally compact subset of a Banach space is compact if and only if it contains no half-line $\left[17\right.$, p.340]. Therefore, $K_{s}$ is compact. Let $s_{0}$ be a fixed number such that $K_{s_{0}}$ is nonempty. Then the family $\left\{K_{s}: s \leqslant s_{0}\right.$ and $\left.K_{s} \neq \emptyset\right\}$ of closed sets has a nonempty intersection. This implies that there exists a smallest number $s^{*} \leqslant s_{0}$ such that $K_{s^{*}}$ is nonempty. It follows that any point in $K_{s^{*}}$ is a nearest point in $C(x)$ to $x_{0}$. Define $U(x)$ to be the distance from one of these points to $x_{0}$. Then the function $U$ is well-defined. 
For each $x \in C\left(x_{0}\right)$, let $M(x)=\left\{x^{\prime} \in C(x):\left\|x_{0}-x^{\prime}\right\|=U(x)\right\}$. By the above argument, $M(x)$ is nonempty for all $x \in C\left(x_{0}\right)$. By using the lower continuity of $\preceq$ and convexity of $X$ it is easily verified that $x \sim x^{\prime}$. This implies that $U$ is order preserving on $C\left(x_{0}\right)$.

By condition (2) there exists a countable co-initial subset $Z$. Let $Z=\left\{z_{1}, z_{2}, \ldots\right\}$. For each $z_{n} \in Z$ we have an order preserving function $\bar{U}_{n}$ on $C\left(z_{n}\right)$ by the first part of the proof. The Debreu Open Gap Lemma (see [10] or [5, p.44]) implies that there is a continuous order preserving function $U_{n}$ on $C\left(z_{n}\right)$. Since $\mathbb{R}$ is order-homeomorphic to $(0,1)$ we may assume that each $U_{n}$ is bounded. Now define a function $V_{n}$ on $X$ for each $n=1,2, \ldots$ as follows. Let $V_{n}(y)=U_{n}(y)$ for $y \in C\left(z_{n}\right)$ and $V_{n}(y)=U\left(z_{n}\right)$ for $y \in X \backslash C\left(z_{n}\right)$. Then $V_{n}$ is an extension of $U_{n}$ to $X$ which is continuous by the Glueing Lemma $\left[9\right.$, p.151]. Finally, we define a function $V$ on $X$ by $V(x)=\sum_{n=1}^{\infty} 2^{-n} V_{n}(X)$. The function $V$ is clearly isotone and continuous. To prove that $V$ is strictly isotone suppose that $x \prec y$. There exists $z_{n} \in Z$ such that $z_{n} \preceq x$. It follows that for this $n$ we have $V_{n}(x)<V_{n}(y)$ whence we get $V(x)<V(y)$ and the theorem is proved.

EXAMPLE 3. We now give an example of a total preorder that satisfies the conditions of Theorem 3. To that end, we need the following lemma whose proof is omitted for the sake of brevity.

LEMma. Let $E$ be a Banach space and $A \subset E$ a convex and compact subset. Then $X=\left\{t . a: t \in \mathbb{R}_{+}, a \in A\right\}$ is a closed convex and locally compact subset of $E$.

Now consider the Banach space $E=c_{0}$ and define the map:

$$
\lambda \in[1,2] \rightarrow \varphi(\lambda)=\left(\frac{\sqrt[n]{\lambda}}{n}\right)_{n=1}^{\infty} \in c_{0} .
$$

Notice that $\varphi$ is injective and continuous. Define $A=\overline{\mathrm{co}}[\varphi([1,2])$ ) (where $\overline{\operatorname{co}}(B)$ stands for the closed convex hull of $B)$. Since $\varphi([1,2])$ is a compact subset of $c_{0}$, it follows from $[19$, p.61], that $A$ is convex and compact as well. Finally take $X$ as in the above lemma. Then $X \subset c_{0}$ is closed and convex. On $X$ consider the following total preorder $\preceq$ :

$$
\left(x_{n}\right)_{n=1}^{\infty} \preceq\left(y_{n}\right)_{n=1}^{\infty} \text { if and only if } x_{1} \leqslant y_{1} \quad\left(\left(x_{n}\right)_{n=1}^{\infty},\left(y_{n}\right)_{n=1}^{\infty} \in X\right) .
$$

Then $\preceq$ satisfies all the conditions of Theorem 3 .

Local compactness is a very stringent assumption in Banach spaces. However, it should be noted that our approach is based on the existence of elements with the best approximation property. In connection with this point it should be ntoed that this last property forces the space to have a special linear structure. Indeed, it is well-known 
that a Banach space is reflexive if and only if each closed hyperplane is proximal [24, p.99]. This does not mean that the Banach space in Theorem 3 is reflexive. Rather if $E$ is a Banach space such that each closed hyperplane is proximal then $E$ is reflexive.

As an illustration of the previous paragraph one can easily prove that the hyperplane $H=\left\{\left(x_{n}\right)_{n=1}^{\infty} \in c_{0}: \sum_{n=1}^{\infty}\left(x_{n} / 2^{n}\right)=0\right\}$ is not proximinal in $c_{0}$.

\section{REFERENCES}

[1] A. Araujo, 'Lack of Pareto optimality for economies with infinitely many goods: the need for impatience', Econometrica 53 (1985), 455-461.

[2] A. Araujo, 'A note on the existence of Pareto optima in topological vector spaces', Econom. Lett. 23 (1987), 5-7.

[3] K. Arrow and F. Hahn, General competitive analysis (Oliver and Boyd, Edinburgh, 1971).

[4] T. Bewley, 'Existence of equilibria with infinitely many commodities', J. Econom. Theory 4 (1972), 514-540.

[5] D.S. Bridges and G.B. Mehta, Representations of preference orderings (Springer-Verlag, Berlin, Heidelberg, New York, 1995).

[6] D. Brown and L. Lewis, 'Myopic economic agents', Econometrica 49 (1981), 359-368.

[7] J.C. Candeal and E. Induráin, 'Homothetic and weakly homothetic preferences', J. Math. Econom. 24 (1995), 147-158.

[8] J.C. Candeal, E. Induráin and G.B. Mehta, 'Some utility theorems on inductive limits of preordered topological spaces', Bull. Austral. Math. Soc. 52 (1995), 235-246.

[9] F. Croom, Principles of topology (Saunders College Publishing, 1989).

[10] G. Debreu, 'Representation of a preference ordering by a numerical function', in Decision processes, (R. Thrall, C. Coombs and R. Davis, Editors) (Wiley, New York, 1954), pp. $159-166$.

[11] J. Dugundji, Topology, Advanced Mathematics (Allyn and Bacon Inc., Boston, MA, 1978).

[12] S. Eilenberg, 'Ordered topological spaces', Amer. J. Maths. 63 (1941), 39-45.

[13] M. Estévez and C. Hervés, 'On the existence of continuous preference orderings without utility representations', J. Math. Econom. 24 (1995), 305-309.

[14] P. Fishburn, 'Utility functions on ordered convex sets', J. Math. Econom. 12 (1983), 221-232.

[15] G. Herden, 'Topological spaces for which every continuous total preorder can be represented by a continuous utility function', Math. Social Sci. 22 (1991), 123-135.

[16] R. Holmes, Geometrid functional analysis and its applications (Springer-Verlag, Berlin, Heidelberg, New York, 1975).

[17] G. Kothe, Topological vector spaces (Springer-Verlag, Berlin, Heidelberg, New York, 1969).

[18] G.B. Mehta and P.K. Monteiro, 'Infinite dimensional utility representation theorems', Econom. Lett. 53 (1996), 169-173.

[19] P.K. Monteiro, 'Some results on the existence of utility functions on path connected spaces', J. Math. Econom. 16 (1987), 147-156. 
[20] L. Narici and E. Beckenstein, Topological vector spaces (Marcel Dekker, New York, 1985).

[21] J. Ostroy, 'On the existence of Walrasian equilibrium in large-square economies', J. Math. Econom. 13 (1984), 143-163.

[22] W. Rudin, Functional analysis, International Series in Pure and Applied Mathematics (McGraw-Hill, New York, 1991).

[23] W. Shafer, 'Representations of preorders on normed spaces', University of Southern California (1984).

[24] I. Singer, Best approximation in normed linear spaces by elements of linear subspaces (Springer-Verlag, Berlin, Heidelberg, New York).

[25] C. Swartz, Introduction to functional analysis, Monographs and Textbooks in Pure and Applied Mathematics 157 (Marcel Dekker, New York, 1992).

Universidad de Zaragoza

Departamento de Análisis Económico

Facultad de Ciencias Económicas y Empresariales 50005 Zaragoza

Spain

University of Queensland

Department of Economics

Queensland 4072

Australia
Universidad Pública de Navarra

Departamento de Matemáticas e Informática

Campus Arrosadia s.n.

E-31006 Pamplona

Spain 